\title{
INTERNAL ELECTRIC FIELD AND FILL FACTOR OF AMORPHOUS SILICON SOLAR CELLS
}

\author{
Michael Stückelberger ${ }^{1}$, Arvind Shah ${ }^{1}$, Janez $\mathrm{Krc}^{2}$, Matthieu Despeisse ${ }^{1}$, Fanny Meillaud ${ }^{1}$, Christophe Ballif \\ ${ }^{1}$ Ecole Polytechnique Fédérale de Lausanne (EPFL), Institute of Microengineering (IMT), \\ Photovoltaics and Thin Film Electronics Laboratory, Rue A.-L. Breguet 2, CH-2000 Neuchâtel, Switzerland \\ ${ }^{2}$ University of Ljubljana, Faculty of Electrical Engineering, Trzaska 25, Si-1000 Ljubljana, Slovenia \\ Email: michael.stuckelberger@epfl.ch
}

\begin{abstract}
The electric field $E$ within the $i$-layer of hydrogenated amorphous silicon (a-Si:H) solar cells strongly affects the cell performances, and, specifically, the fill factor $F F$. It governs the drift length $L_{\text {drift }}=\mu t E$ which is the crucial parameter limiting charge collection. Ideally, a constant electric field is assumed across the $i$-layer, whereas in real devices, it is deformed by charged band tail states and dangling bonds. If the $i$-layer is too thick or has a high density of charged defects, $E$ is deformed and reduced. To determine theoretically the charge states of band tails and dangling bonds, we must know the carrier density profiles within the $i$-layer. Here, the SunShine program is used to determine carrier generation profiles within $i$-layers of pincells on TCO-covered glass substrates. A classical model for transport and electron/hole capture is employed to determine charge conditions of band tail states and dangling bonds. Results are: (a) charged dangling bonds are predominant for the electric field deformation, affecting the output performance of the cell; (b) this effect is very pronounced especially in degraded cells; (c) it is independent of light intensity; (d) it accounts for performance breakdown of thick, degraded a-Si:H cells. Calculated results are confronted with experimental observations (measurements of $F F$, collection voltage $V_{\text {coll }}$ and external quantum efficiency $E Q E$ ) on pin-type solar cells of $100,200,300$, and $400 \mathrm{~nm}$ thickness produced at IMT Neuchâtel, in initial and degraded state. $L_{\text {drift }}$ is evaluated via $V_{\text {coll }}$, determined here with the method of variable intensity measurements (VIM). Trends observed are explained to full satisfaction.
\end{abstract}

\section{INTRODUCTION}

It is of common knowledge that the internal electric field $E$ in the intrinsic (i) layer is essential for the proper functioning of pin- and nip-type thin-film silicon solar cells. In fact, $E$ determines the collection length (which is here the drift length $L_{\text {drift }}=\mu T E$ ) and governs, thus, the fill factor FF of these cells. Whereas microcrystalline silicon solar cells are generally not seriously affected by collection problems up to $i$-layer thicknesses of about $5 \mu \mathrm{m}$ [1], amorphous silicon (a-Si:H) solar cells have to be kept very thin $(<300 \mathrm{~nm}$ ) to avoid any breakdown of $E$ in the degraded state. The purpose of the present paper is threefold: (1) to propose a very simple model for the approximate calculation of different terms contributing to the field deformation $\Delta E(x)$ within the $i$-layer of a-Si:H solar cells; (2) to present simulation results for pin-type solar cells with $i$-layers of $100,200,300$ and $400 \mathrm{~nm}$ thickness on flat substrates; (3) to compare these numerical results with measurements done on corresponding cells produced at IMT Neuchâtel. Thereby, the following techniques were used to gather experimental data: $J(V)$, VIM (Variable Intensity Measurements) [2], and bifacial EQE (External Quantum Efficiency) measurements [3] varying both bias voltage $V_{\text {bias }}$ and bias light intensity.

\section{SIMPLE THEORETICAL MODEL TO ESTIMATE THE ELECTRIC FIELD DEFORMATION IN THE $i$-LAYER}

In order to illustrate our model of a $300 \mathrm{~nm}$ thick intrinsic a-Si:H layer, we shall first assume:
A. a constant photo-generation rate $G$ throughout the $i$-layer ( $\left.G=2 \times 10^{22} \mathrm{~cm}^{-3} \mathrm{~s}^{-1}\right)$ ("red light").

\section{Free charge carrier profiles within $i$-layer}

To obtain an approximate idea of the free hole and electron concentrations $p_{\mathrm{f}}(x)$ and $n_{\mathrm{f}}(x)$ within the $i$-layer, we simplify further by considering:

\section{B. no recombination within $i$-layer \\ C. carrier transport within i-layer governed only by drift (not by diffusion)}

These assumptions are far from being reached, but are taken as a starting point here to determine the electric field within the $i$-layer and to assess the impacts of the charged band tail states and dangling bonds. The simplifications used will be selectively abandoned in later analysis.

According to assumption $B$ we have linear functions for the fluxes of free holes and electrons $\Phi_{\mathrm{p}}(\mathrm{x})$ and $\Phi_{\mathrm{n}}(\mathrm{x})$ as a function of i-layer thickness, as calculated in equation 1 and shown in figure 1 :

$$
\Phi_{\mathrm{p}}(x)=\int_{x}^{d} G(\xi) \mathrm{d} \xi \text { and } \Phi_{\mathrm{n}}(x)=\int_{0}^{x} G(\xi) \mathrm{d} \xi
$$

Based on assumption $C$, the free hole and electron densities $p_{\mathrm{f}}(x)$ and $n_{\mathrm{f}}(x)$ (see figure 2 ) are obtained via

$$
\begin{aligned}
& p_{\mathrm{f}}=\frac{\Phi_{\mathrm{p}}(x)}{v_{\mathrm{p}}}=\frac{\Phi_{\mathrm{p}}(x)}{\mu_{\mathrm{p}} \cdot E_{\mathrm{nom}}} \\
& n_{\mathrm{f}}=\frac{\Phi_{\mathrm{n}}(x)}{v_{\mathrm{n}}}=\frac{\Phi_{\mathrm{n}}(x)}{\mu_{\mathrm{n}} \cdot E_{\mathrm{nom}}}
\end{aligned}
$$


Here, $v_{\mathrm{p}}$ and $v_{\mathrm{n}}$ are the free carrier velocities, determined in steady-state conditions by the nominal electrical field $E_{\text {nom }}$ and the band mobilities, here considered to be $\mu_{\mathrm{p}}=1 \mathrm{~cm}^{2} /(\mathrm{Vs})$ and $\mu_{\mathrm{n}}=3 \mathrm{~cm}^{2} /(\mathrm{Vs})$, so that

$$
\mu_{\mathrm{n}}=\gamma \cdot \mu_{\mathrm{p}}
$$

with $\gamma=3$. Note that a wide range of values for band mobilities can be found in literature. The nominal electric field is

$$
E_{\text {nom }}=\frac{V_{\text {bi }}}{d}
$$

with $V_{\mathrm{bi}}$ the built-in voltage (taken to be $V_{\mathrm{bi}}=1.1 \mathrm{~V}$ ) and $d$ the thickness of the $i$-layer.

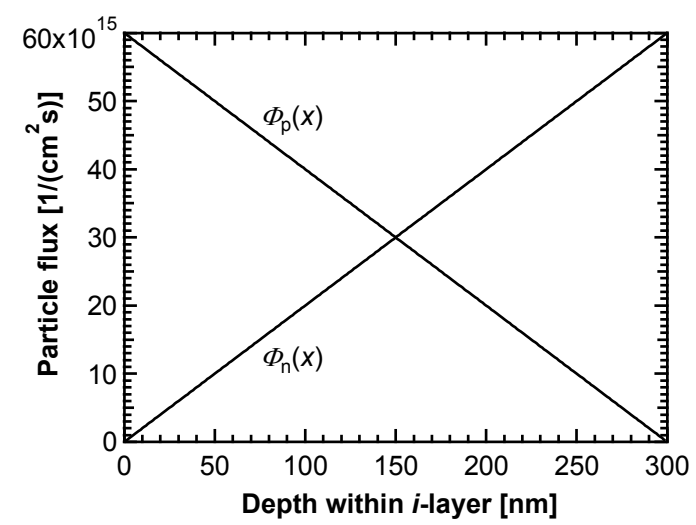

Figure 1 Free hole and electron flux within a $300 \mathrm{~nm}$ thick $i$-layer under the assumptions of constant photogeneration $\left(G=2 \times 10^{22} \mathrm{~cm}^{-3} \mathrm{~s}^{-1}\right)$, no recombination, and only drift-driven charge transport.

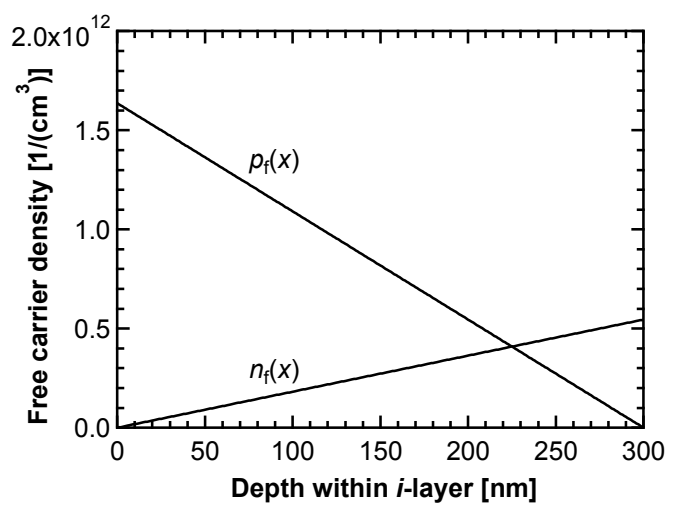

Figure 2 Free hole and electron density profiles; These are not symmetrical due to the assumption of different mobilities in a-Si:H $\left(\mu_{n}=3 \mu_{p}\right)$.

\section{Trapped charge carrier profiles within the $\boldsymbol{i}$-layer}

The concentrations of charge carriers $p_{\mathrm{t}}(x)$ and $n_{\mathrm{t}}(x)$ that are trapped in band tail states are assumed to be proportional to the free carrier concentrations $p_{\mathrm{f}}(x)$ and $n_{\mathrm{f}}(x)$ :

$$
p_{\mathrm{t}}=\frac{p_{\mathrm{f}}}{\Theta_{\mathrm{p}}} \text { and } n_{\mathrm{t}}=\frac{n_{\mathrm{f}}}{\Theta_{\mathrm{n}}},
$$

where the "Rose trapping" factors $\Theta_{\mathrm{p}}$ and $\Theta_{\mathrm{n}}$ are set as $\Theta_{p}=0.005$ and $\Theta_{\mathrm{n}}=0.1$. Note that choosing (within a reasonable range) different values for the Rose trapping factors as well as for the band mobilities will affect the results only quantitatively but not qualitatively. Figure 3 shows the trapped charge carrier profiles $p_{\mathrm{t}}(x)$ and $n_{\mathrm{t}}(x)$ for the example presented in figures 1 and 2 . Due to the large ratio $\Theta_{\mathrm{n}} / \Theta_{\mathrm{p}}$ it is obvious that $p_{\mathrm{t}}(x) \gg n_{\mathrm{t}}(x)$. Therefore, the contribution of trapped electrons contribution is neglected from now on.

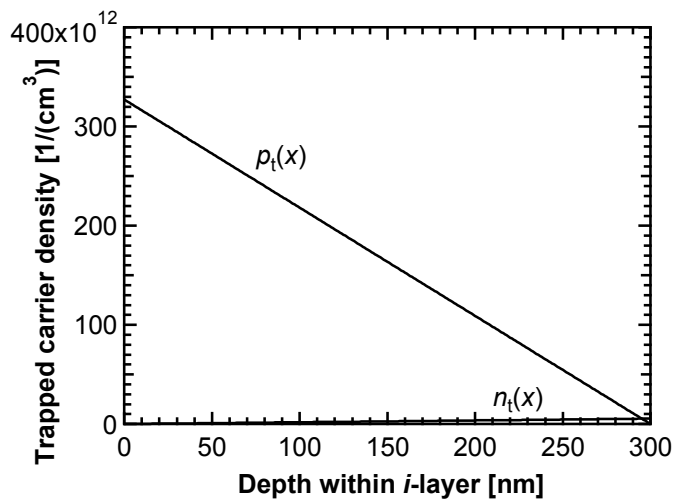

Figure 3 Trapped charge carrier profiles $p_{t}(x)$ and $n_{t}(x)$, within $i$-layer of pin-type solar cell, according to equation (5), and as long as assumptions A, B, C (see above) can be upheld. This example refers to an i-layer thickness of $300 \mathrm{~nm}$.

\section{Electric field deformation due to charged band tails}

As a very first assumption, the electric field is supposed to be constant within the $i$-layer according to equation 4 . However, this field is shielded by the space charge of free and trapped holes and electrons that lead to a depth dependent electric field deformation $\Delta E_{\mathrm{bt}}(x)$ due to charged band tails. In practice, it is entirely sufficient to consider only the trapped holes, as all other charge carrier concentrations $p_{\mathrm{f}}, n_{\mathrm{f}}$, and $n_{\mathrm{t}}$ are far lower than $p_{\mathrm{t}}$, as can be seen in figures 2 and 3 . Considering only the trapped holes, the space charge is

$$
\rho_{\mathrm{bt}}(x)=q \cdot p_{\mathrm{t}}(x)
$$

with the elementary charge $q=1.602 \times 10^{-19} \mathrm{C}$. Still holding all assumptions made so far, the field deformation due to charged dangling bonds can then be described using Poissons's equation:

$$
\Delta E_{\mathrm{bt}}(x)=\frac{1}{\varepsilon_{0} \varepsilon_{\mathrm{r}}} \int_{0}^{x} \rho_{\mathrm{bt}}(\xi) \mathrm{d} \xi,
$$

where $\varepsilon_{0}=8.854 \times 10^{-14} \mathrm{~F} / \mathrm{cm}$ and $\varepsilon_{r} \approx 10$. Figure 4 shows this field deformation as a function of the position in the $i$ layer. Please note that electric field deformation is always a shielding effect that reduces the nominal electric field. However, it is plotted as a positive quantity here. 


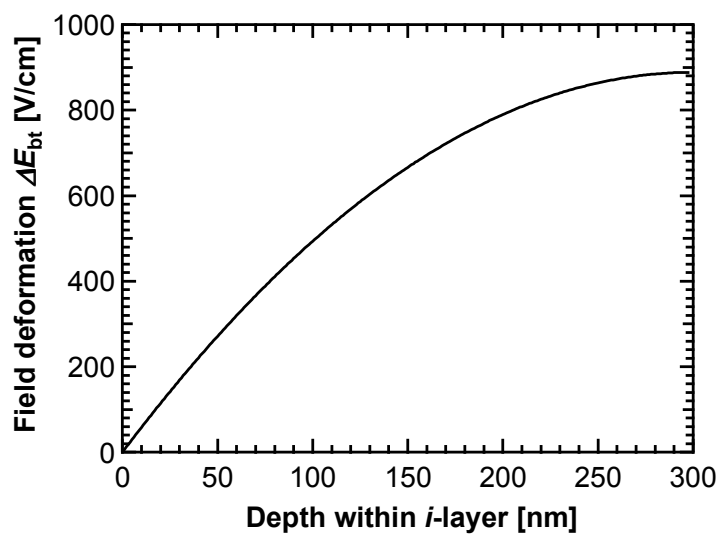

Figure 4 Field deformation caused by charged band tail states. Only trapped holes are considered here, as trapped and free electrons, as well as free holes hardly contribute to field deformation due to their much smaller concentrations.

\section{Field deformation due to charged dangling bonds}

Not only charged band tail states as described above but also charged dangling bonds lead to an electric field deformation within the $i$-layer. To estimate this contribution $\Delta E_{\mathrm{db}}(x)$ we assume here:

a. amphoteric dangling bonds (here the three charge states $D^{+}, D^{0}, D^{-}$)

b. that the occupation of the dangling bond states is determined by the competition of the four capture processes with rates $r_{\mathrm{a}}, r_{\mathrm{b}}, r_{\mathrm{c}}$, and $r_{\mathrm{d}}$ as shown in figure 5 .

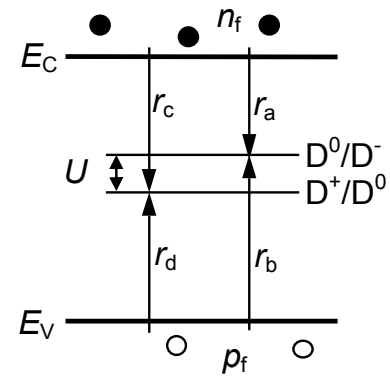

Figure 5 Capture of free holes $p_{\mathrm{f}}$ and free electrons $n_{\mathrm{f}}$ into charged $\mathrm{D}^{+}, \mathrm{D}^{-}$, and uncharged dangling bond states. Thermal generation is not shown (it can be neglected for sufficiently illuminated solar cells). $U$ is the so-called "correlation energy" or "Hubbard energy".

Capture processes with rates $r_{\mathrm{b}}$ and $r_{\mathrm{c}}$ are charge-assisted and supposed to have the same cross section $\sigma$. Also, they can be assumed to be much larger than the capture processes of neutral states with rates $r_{\mathrm{d}}$ and $r_{\mathrm{a}}$ [4]:

$$
\begin{gathered}
\sigma_{r_{\mathrm{b}}} \equiv \sigma_{\mathrm{p}}^{-}=\sigma^{ \pm}=\sigma_{\mathrm{n}}^{+} \equiv \sigma_{r_{\mathrm{c}}} \\
\sigma_{r_{\mathrm{d}}} \equiv \sigma_{\mathrm{p}}^{0}=\sigma^{0}=\sigma_{\mathrm{n}}^{0} \equiv \sigma_{r_{\mathrm{a}}} \\
\sigma^{ \pm}=\zeta \cdot \sigma^{0}
\end{gathered}
$$

For further calculations we assume $\zeta=50$ as proposed in [5] and introduce, in extension of [4] and in analogy to equation 3 , different thermal velocities for electrons and holes, such that

$$
v_{n}^{\text {th }}=\gamma \cdot v_{p}^{\text {th }},
$$

with $\gamma=3$. Further, we define

$$
\chi(x) \equiv \gamma \frac{n_{f}(x)}{p_{f}(x)} .
$$

Due to assumption C, $\chi(x)=\Phi_{n} / \Phi_{p}$ is valid here. Based on capture kinetics, as detailed in [4], but with the above additional distinction between thermal electron and hole velocities (9), we can divide numerator and denominator of equation 8 [4] by $\left[\left(v_{p}^{\text {th }}\right)^{2} \sigma^{ \pm} \sigma^{0} p_{f}^{2}\right]$ and rewrite the occupation functions of dangling bond states with the new symbols as

$$
f^{+}=\frac{1}{1+\zeta \chi+\chi^{2}}, \quad f^{0}=\frac{\zeta \chi}{1+\zeta \chi+\chi^{2}}, \quad f^{-}=\frac{\chi^{2}}{1+\zeta \chi+\chi^{2}}
$$

For the simulation we simplify that dangling bonds are in the state

$$
\begin{aligned}
& \mathrm{D}^{+}, \text {if } f^{+}>f^{0} \text { or } \chi<\zeta^{-1} \\
& \mathrm{D}^{0}, \text { if } f^{0}>f^{+}, f^{-} \text {or } \zeta \geq \chi \geq \zeta^{-1} \\
& \mathrm{D}^{-}, \text {if } f^{-}>f^{0} \text { or } \chi>\zeta
\end{aligned}
$$

Taking the free hole and electron fluxes from equation 1 or figure 1 , one can now determine the thicknesses $d^{+}$and $d^{\prime}$ of the regions where the dangling bonds are completely charged as shown in figure 6 , where $\zeta=10$ has been chosen for illustration.

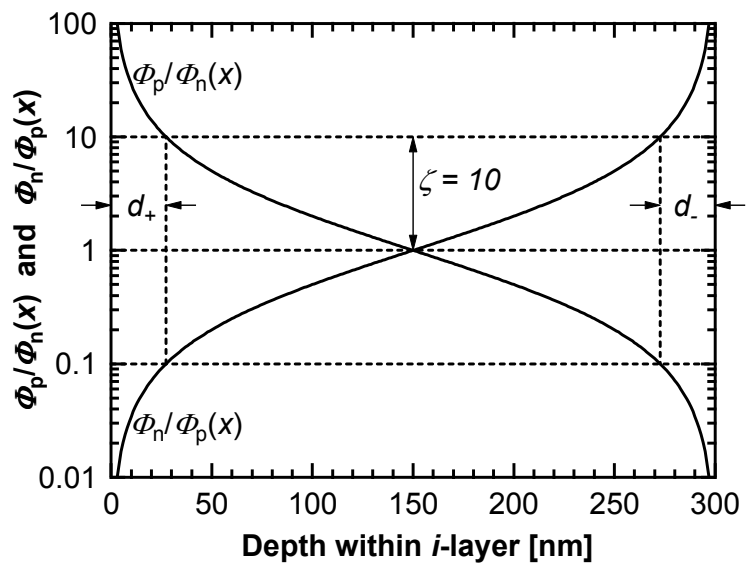

Figure 6 Ratios $\chi=\Phi_{n} / \Phi_{p}$ and $\chi^{-1}=\Phi_{p} / \Phi_{n}$ of free carriers for the example mentioned above with a constant electron/hole pair generation rate over the whole $i$-layer of a pin-type solar cell. Dangling bonds within distances $d^{+}$and $d$ from the $p$ - and $n$-layer, respectively, are considered to be completely charged. Although $\zeta=50$ has been used for calculations, it has been set to 10 for illustration here. 
In analogy to the electric field deformation due to charged band tail states, the electric field deformation due to charged dangling bonds can therefore be calculated via adapted equations 6 and 7 for the two regions with charged dangling bonds. In this simple model, where $\rho_{\mathrm{db}}(x)$ is a step function, the field deformation due to charged dangling bonds is constant within the i-layer except near the $p / i$ - and $i / n$-interfaces where it is linear.

\section{NUMERICAL EXAMPLES FOR EVALUATING THE ELECTRIC FIELD DEFORMATION}

Now we consider a more realistic case: a pin-type solar cell structure as indicated in figure 7 , illuminated with AM1.5g $\left(1000 \mathrm{~W} / \mathrm{m}^{2}\right)$ from $p$-side.

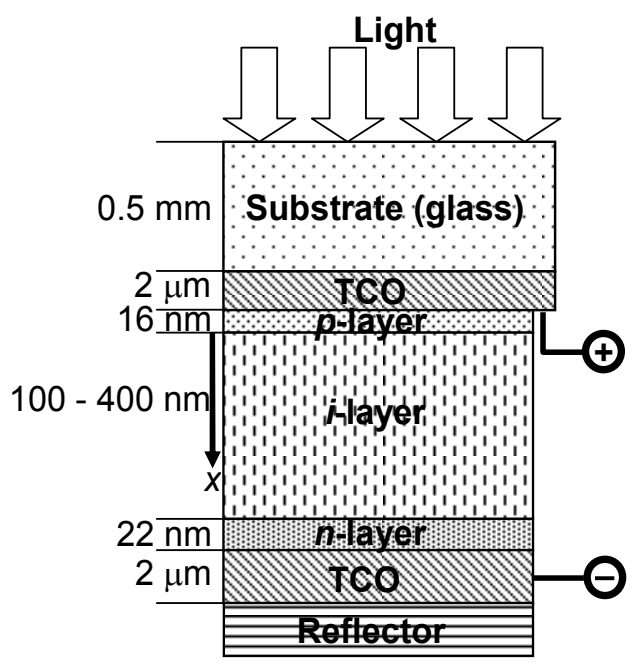

Figure 7 Structure of the amorphous silicon pin solar cell deposited with PE-CVD on flat TCO (ZnO) used for numerical simulation.

The photo-generation profile in the $i$-layer of the presented structure was calculated with the help of the SunShine program [6]. Thereafter, the same procedure was applied as explained in detail in section 2 above. This was done for the cells with $i$-layer thicknesses of 100, 200,300, and $400 \mathrm{~nm}$. As an example, the flux ratios of free electrons and holes that lead to electric field deformation due to charged dangling bonds are shown in figure 8.

Table 1 shows the maximum reduction $\Delta E_{\mathrm{bt}}$ of the internal electric field $E_{\text {nom }}$ due to trapped holes in the valence band tail. The following numerical values have been assumed for the calculation: built-in voltage $V_{\mathrm{bi}}=1.1 \mathrm{~V}$, hole band mobility $\mu_{p}=1 \mathrm{~cm}^{2} /(\mathrm{Vs}), \Theta_{\mathrm{p}}=0.005$. Contributions of trapped electrons and free electrons and holes have been neglected (it has been checked with $\mu_{\mathrm{n}}=3 \mathrm{~cm}^{2} /(\mathrm{Vs})$ and $\Theta_{\mathrm{n}}=0.1$ that their effect is indeed by orders of magnitude smaller compared to trapped holes).

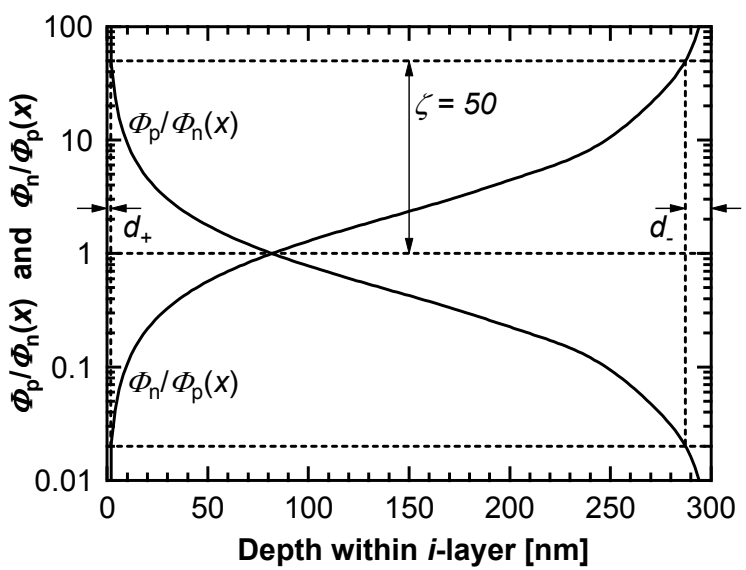

Figure 8 Ratios $\chi=\Phi_{\mathrm{n}} / \Phi_{\mathrm{p}}$ and $\chi^{-1}=\Phi_{\mathrm{p}} / \Phi_{\mathrm{n}}$ of free carriers resulting from a SunShine simulation of a photo-generation rate in a $300 \mathrm{~nm}$ thick a-Si:H solar cell deposited in pin structure on flat $\mathrm{ZnO}$. They are used to calculate the electric field deformation due to charged dangling bonds within $d^{+}$and $d$.

\begin{tabular}{cccc}
\hline$d$ & $E_{\text {nom }}$ & $\max \left(\Delta E_{\mathrm{bt}}\right)$ & $\max \left(\Delta E_{\mathrm{bt}} / E_{\mathrm{nom}}\right)$ \\
\hline \hline $100 \mathrm{~nm}$ & $1.1 \times 10^{5} \mathrm{~V} / \mathrm{cm}$ & $8.2 \times 10^{1} \mathrm{~V} / \mathrm{cm}$ & $0.1 \%$ \\
$200 \mathrm{~nm}$ & $5.5 \times 10^{4} \mathrm{~V} / \mathrm{cm}$ & $3.6 \times 10^{2} \mathrm{~V} / \mathrm{cm}$ & $0.7 \%$ \\
$300 \mathrm{~nm}$ & $3.7 \times 10^{4} \mathrm{~V} / \mathrm{cm}$ & $8.2 \times 10^{2} \mathrm{~V} / \mathrm{cm}$ & $2.3 \%$ \\
$400 \mathrm{~nm}$ & $2.8 \times 10^{4} \mathrm{~V} / \mathrm{cm}$ & $1.5 \times 10^{3} \mathrm{~V} / \mathrm{cm}$ & $5.3 \%$ \\
\hline
\end{tabular}

Table 1 Simulated maximal reduction of internal electric field due to trapped holes for different $i$-layer thickness $d$

In order to estimate the electric field deformation due to charged dangling bonds, we need to make the following additional assumptions: Ratio $\mu_{\mathrm{n}}: \mu_{\mathrm{p}}=v_{\mathrm{n}}^{\text {th }}: v_{\mathrm{p}}^{\text {th }}=\gamma=3$, $\sigma_{\mathrm{n}}^{+}: \sigma_{\mathrm{n}}^{0}=\sigma_{\mathrm{p}}^{-}: \sigma_{\mathrm{p}}^{0}=\zeta=50$, dangling bond density $N_{\mathrm{db}}=$ $2 \times 10^{16} \mathrm{~cm}^{-3}$. Tables 2 show the results obtained with these parameters.

\begin{tabular}{|c|c|c|c|}
\hline$d$ & $d_{+}$ & $\max \left(\Delta E_{+\mathrm{db}}\right)$ & $\max \left(\Delta E_{+\mathrm{db}} / E_{\mathrm{nom}}\right)$ \\
\hline $100 \mathrm{~nm}$ & $1.2 \mathrm{~nm}$ & $4.3 \times 10^{2} \mathrm{~V} / \mathrm{cm}$ & $0.4 \%$ \\
\hline $200 \mathrm{~nm}$ & $1.7 \mathrm{~nm}$ & $6.0 \times 10^{2} \mathrm{~V} / \mathrm{cm}$ & $1.1 \%$ \\
\hline $300 \mathrm{~nm}$ & $1.9 \mathrm{~nm}$ & $6.9 \times 10^{2} \mathrm{~V} / \mathrm{cm}$ & $1.9 \%$ \\
\hline $400 \mathrm{~nm}$ & $2.0 \mathrm{~nm}$ & $7.4 \times 10^{2} \mathrm{~V} / \mathrm{cm}$ & $2.7 \%$ \\
\hline$d$ & $d$ & $\max \left(\Delta E_{-\mathrm{db}}\right)$ & $\max \left(\Delta E_{-\mathrm{db}} / E_{\mathrm{nom}}\right)$ \\
\hline $100 \mathrm{~nm}$ & $3.3 \mathrm{~nm}$ & $1.2 \times 10^{3} \mathrm{~V} / \mathrm{cm}$ & $1.1 \%$ \\
\hline $200 \mathrm{~nm}$ & $7.3 \mathrm{~nm}$ & $2.6 \times 10^{3} \mathrm{~V} / \mathrm{cm}$ & $4.8 \%$ \\
\hline $300 \mathrm{~nm}$ & $13 \mathrm{~nm}$ & $4.6 \times 10^{3} \mathrm{~V} / \mathrm{cm}$ & $13 \%$ \\
\hline $400 \mathrm{~nm}$ & $19 \mathrm{~nm}$ & $6.9 \times 10^{3} \mathrm{~V} / \mathrm{cm}$ & $25 \%$ \\
\hline
\end{tabular}

Tables 2 Simulated maximal reduction of internal electric field due to charged dangling bonds for different $i$-layer thickness. Upper table: positively charged dangling bonds near the $p / i$ interface. Lower table: negatively charged dangling bonds at the $i / n$ interface. 
As conclusion of this section 3, we can state:

- Deformation of the internal electric field due to charged dangling bonds is in general slightly more pronounced than that due to trapped holes in the valence band tail.

- For illumination from $p$-side, the dangling bond space charge at the p/i-interface is smaller than the space charge at the $i / n$-interface, so that negatively charged dangling bonds are most responsible of the field deformation.

- From other simulation results (not shown here) we have been able to verify that the roughness (texture) of the $\mathrm{ZnO}$ (TCO) layer has only a small effect on the various space charge contributions.

\section{EXPERIMENTAL RESULTS}

According to the simulated cells described in section 3 above, pin-type a-Si:H solar cells with $i$-layer thicknesses of $100,200,300$, and $400 \mathrm{~nm}$ and a structure as given in figure 7 were deposited. Unfortunately, these cells have strong boron tailing. Several measurements have been carried out on the cells before and after light induced degradation:

- VIM (Variable Intensity Measurement, [2]): J(V) curves were measured under an AM1.5g solar simulator using grey filters letting pass $0.4,0.76$, $10.4,26,50$ and $100 \%$ of the total irradiance equivalent to $1000 \mathrm{~W} / \mathrm{m}^{2}$.

- Bifacial EQE (External Quantum Efficiency, [3]): Illuminating the cells from the $p$-side with $0,26,50$ and $100 \%$ of $A M 1.5 \mathrm{~g}$ bias light $\left(1000 \mathrm{~W} / \mathrm{m}^{2}\right)$, the $E Q E$ has been measured with the frequency modulated probe light from the $p$ - and from the $n$ side. Bias voltages of $-1.5,-1,-0.5,-0.25,0,0.15$, 0.3 and $0.5 \mathrm{~V}$ were applied for these measurements.

\section{Collection voltage measurement}

From VIM, the fill factor $F F$ and the collection voltage $V_{\text {coll }}$ were determined via the short-circuit resistance $R_{\mathrm{sc}}$ and the short-circuit current density $J_{\mathrm{sc}}$ as described in [2]. Figure 9 shows such a measurement from which $V_{\text {coll }}$ could be determined as

$$
V_{\text {coll }}=R_{\mathrm{sc}} \cdot J_{\mathrm{sc}}
$$

from the linear part of the curve at high illumination (i.e. high currents) and the shunt resistance $R_{\text {shunt }}$ from the plateau at low illumination. The resulting collection voltages and fill factors are shown in figure 10. As is expected, $V_{\text {coll }}$ and $F F$ decrease in approximately the same order of magnitude with increasing $i$-layer thickness, for which charge collection gets worse due to lower nominal electric field $E_{\text {nom }}$ and larger electric field deformation as shown in the previous section. The degradation of $V_{\text {coll }}$ and $F F$ can be attributed to a lightinduced increase of the dangling bond density $N_{\mathrm{db}}$.

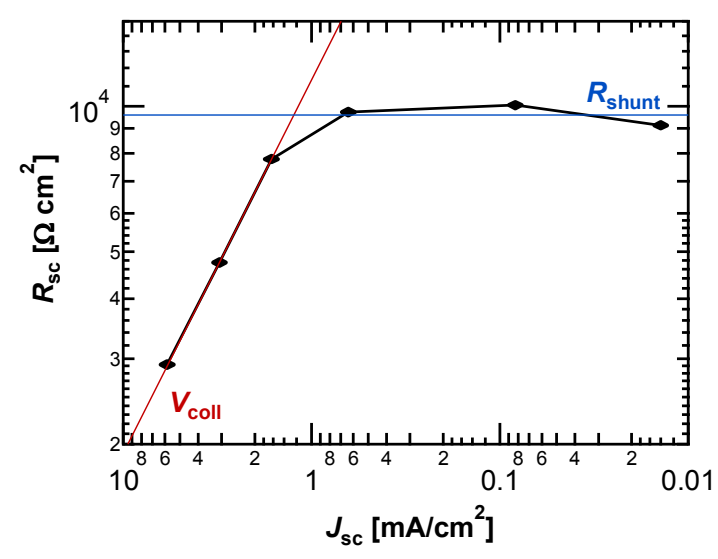

Figure 9 Short-circuit resistance $R_{\mathrm{sc}}$ as a function of the short-circuit current density $J_{\mathrm{sc}}$ extracted from variable intensity measurements. The collection voltage $V_{\text {coll }}$ was determined from the linear part of the curve at high illumination and the shunt resistance $\boldsymbol{R}_{\text {shunt }}$ from the plateau at low illumination.

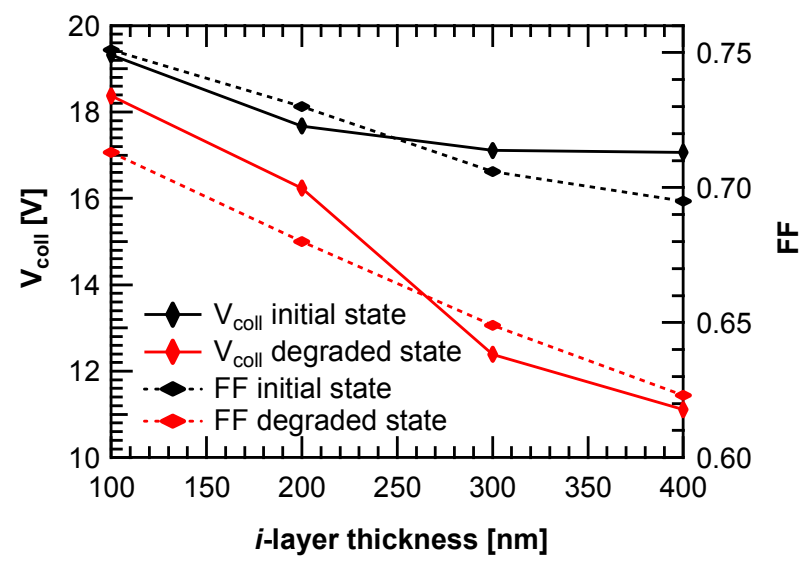

Figure 10 Collection voltage $V_{\text {coll }}$ and fill factor $F F$ for cells with $100,200,300$, and $400 \mathrm{~nm}$ thick $i$-layers before and after degradation. $V_{\text {coll }}$ and $F F$ are measured at $1000 \mathrm{~W} / \mathrm{m}^{2} \mathrm{AM} 1.5 \mathrm{~g}$ illumination.

\section{Bifacial EQE measurements}

Normalized EQE measurements as a function of the bias voltage are shown for different bias light intensities in figures 11 (probe light from $p$-side) and 12 (probe light from $n$-side). 


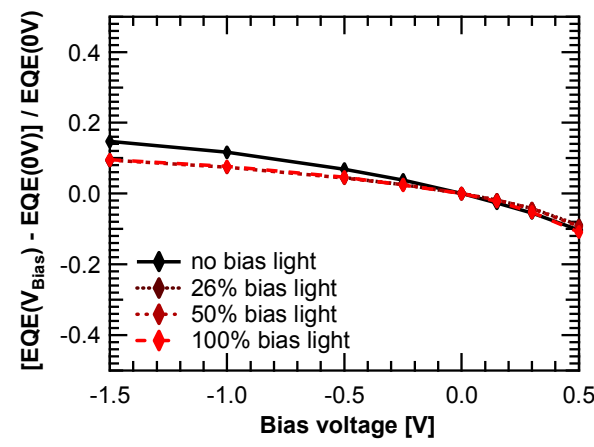

Figure 11 Normalized external quantum efficiency of the $300 \mathrm{~nm}$ thick degraded cell as a function of the bias voltage for different bias light intensities. Both bias light and probe light at $420 \mathrm{~nm}$ were applied from the $p$-side. Therefore, this measurement is sensitive mainly to phenomena near the p/i-interface.

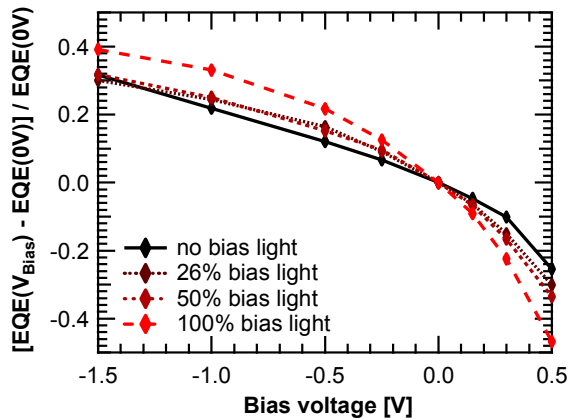

Figure 12 Normalized external quantum efficiency of the $300 \mathrm{~nm}$ thick degraded cell as a function of the bias voltage for different bias light intensities. While bias light was applied from $p$-side, the probe light at $420 \mathrm{~nm}$ entered the cell from the $n$-side. Therefore, this measurement is sensitive mainly to phenomena near the iln-interface.

\section{DISCUSSION}

From the simulations in section 3 and from the experimental measurements in section 4 we note that:

- The electric field in the $i$-layer is mainly affected by negatively charged dangling bonds at the i/ninterface. This is clearly seen in the simulation results. As for the experimental results: a comparison of Figures 11 and 12 indicates also that $i / n$-interface is more strongly affected by degradation than the $p / i-$ interface.

- The electric field in the $i$-layer is affected only to a much lesser extent by charges trapped in the valence band tail. This again is clearly seen by the simulation results. As for the experimental results, we note a slight dependency of $\triangle E Q E / E Q E(0 \mathrm{~V})$ at $420 \mathrm{~nm}$ wavelength on bias light: this shows up in figure 12 , but not in figure 11. This can be taken as indication that the space charge due to holes trapped in the valence band tail contributes only marginally to the deformation of the electric field.
- The measured values of the collection voltage $V_{\text {coll }}$ are clearly affected by degradation. However their dependency on $i$-layer thickness is smaller than expected. The experiment therefore needs to be repeated on a thickness series of pin-solar cells which do not exhibit boron tailing.

\section{CONCLUSIONS}

A simple model to estimate the effect of space charge within the $i$-layer of pin solar cells has been proposed. Numerical simulations based on this model and on the optical simulation program SunShine have been carried out. They indicate that the main contribution to the deformation of the electric field in the degraded state is due to space charge of negatively charged dangling bonds near the $i / n$-interface and not (as one would generally expect) due to space charge of positively charged dangling bonds near the $p / i$-interface. This observation should be supported by further measurements on another set of cells. In some cases space charge due to holes trapped in the valence band tail may also play a role. If these conclusions are found to be generally valid one should proceed to examine the consequence of our findings on the design and optimization of solar cells.

\section{ACKNOWLDEDGMENTS}

We acknowledge Swiss Federal Office of Energy (SFOE) for financial support.

\section{REFERENCES}

[1] M. Boccard, et al., "Substrate dependent stability and interplay between optical and electrical properties in $\mu \mathrm{c}-$ Si:H single junction solar cells", Sol. Energy Mater. Sol. Cells, 2010, doi:10.1016/j.solmat.2010.04.043

[2] A.V. Shah, et al., "Diagnostics of thin-film silicon solar cells and solar panels/modules with variable intensity measurements (VIM)", Sol. Energy Mater. Sol. Cells, 2010, doi:10.1016/j.solmat.2010.04.070

[3] D. Fischer, et al., "Amorphous silicon solar sells with graded low-level doped i-layers characterised by bifacial measurements", Proceedings of the $23^{\text {rd }}$ IEEE Photovoltaic Specialists Conference, Louisville, 1993, pp. 878-884

[4] J. Hubin, A.V. Shah, E. Sauvain, "Effects of dangling bonds on the recombination function in amorphous semiconductors", Philosophical Magazine Letters 66, 1992, pp. $115-125$

[5] N. Beck, N. Wyrsch, Ch. Hof, A. Shah, "Mobility lifetime product - A tool for correlating a-Si:H film properties and solar cell performances", J. Appl. Phys. 79, No. 12, 1996

[6] J. Krc, F. Smole, M. Topic, "Analysis of light scattering in amorphous $\mathrm{Si}: \mathrm{H}$ solar cells by a one-dimensional semicoherent optical model", Progress in photovoltaics 11, 2003, pp. 15-26 